\title{
Circular RNA sequencing reveals the molecular mechanism of the effects of acupuncture and moxibustion on endometrial receptivity in patients undergoing infertility treatment
}

\author{
JIE SHEN $^{1 *}$, LI CHEN $^{2 *}$, JIE CHENG $^{1}$, XUN JIN $^{1}$, YANYUN MU $^{1}$, QIAN LI $^{1}$, \\ LIANGJUN XIA ${ }^{3}$, YOULING GAO ${ }^{1}$ and YOUBING XIA ${ }^{1,4}$
}

\begin{abstract}
${ }^{1}$ The Second Clinical College, Nanjing University of Chinese Medicine, Nanjing, Jiangsu 210023; ${ }^{2}$ Reproductive Medical Center of Nanjing Jinling Hospital and The Collaborative Innovation Platform for Reproductive Biology and Technology, Nanjing University School of Medicine, Nanjing, Jiangsu 210002; ${ }^{3}$ School of Medicine and Life Sciences, Nanjing University of Chinese Medicine, Nanjing, Jiangsu 210023; ${ }^{4}$ The First Clinical College, Nanjing Medical University, Nanjing, Jiangsu 211166, P.R. China
\end{abstract}

Received September 7, 2018; Accepted May 20, 2019

DOI: $10.3892 / \mathrm{mmr} .2019 .10386$

\begin{abstract}
The present study aimed to determine the profile of differentially expressed circular RNAs (circRNAs) in infertile patients treated with acupuncture and moxibustion and verify the role of acupuncture and moxibustion in altering endometrial receptivity (ER). High-throughput RNA sequencing and bioinformatics analysis of samples from six pairs of patients treated with or without acupuncture and moxibustion were conducted. The reliability of high-throughput RNA sequencing was validated using reverse transcription-quantitative PCR. The most significant circRNA functions and pathways were selected by gene ontology (GO) and Kyoto Encyclopedia of Genes and Genomes (KEGG) analyses. A circRNA-miR-mRNA interaction network was constructed to determine the connection between circRNAs, microRNAs (miRs), and mRNAs. High-throughput RNA sequencing identified 2,653 circRNAs. A total of 86 circRNAs was differentially expressed, of which 57 were upregulated and 29 were downregulated, between the acupuncture and moxibustion group and the control group. In the GO analysis, the identified BP terms were chromatin modification, positive regulation of transcription from RNA polymerase II promoter involved in unfolded protein response, oxidative DNA demethylation, regulation of transcription from RNA polymerase II promoter in response to hypoxia, and regulation of smooth muscle cell differentiation. The identified CC terms were nucleoplasm, nucleolus, nucleus,
\end{abstract}

Correspondence to: $\mathrm{Dr}$ Youbing Xia, The Second Clinical College, Nanjing University of Chinese Medicine, 138 Xianlin Avenue, Nanjing, Jiangsu 210023, P.R. China

E-mail: xybd1968@sina.com

${ }^{*}$ Contributed equally

Key words: acupuncture, ER, circRNA, high-throughput sequencing histone acetyltransferase complex, and annulate lamellae. The identified MF terms were methylcytosine dioxygenase activity, chromatin binding, zinc ion binding, histone binding, and protein binding. In the KEGG pathway analysis, the identified pathways were protein processing in endoplasmic reticulum, degradation of aromatic compounds, shigellosis, mTOR signaling pathway, bacterial invasion of epithelial cells, and prostate cancer. Circ-SFMBT2, circ-BACH1, and circ-LPAR1 were significantly upregulated $(\mathrm{P}<0.05)$ and associated with numerous miRs and mRNAs. Acupuncture and moxibustion could impact ER by regulating the expression of circRNAs.

\section{Introduction}

Currently, increasing families are facing infertility problems. It has been estimated that 186 million people are affected by infertility, imposing a heavy burden on both families and society (1). With the development of assisted reproductive technology (ART), especially IVF-embryo transfer (IVF-ET), the pregnancy rate has significantly increased during the past decades (2). However, there are still many factors that lead to implantation failure.

To establish a successful implantation, a developmentally competent embryo and a receptive endometrium are both essential (3). Endometrial receptivity (ER) changes with the menstrual cycle, and an embryo can only be implanted during a short period of time called the 'window', when ER is appropriate for implantation (4). ER is a critical factor of gestation and closely related to infertility. Lack of receptivity or an embryo transfer that is not synchronized with a receptive endometrium represents a major reason for implantation failure and the subsequent failure of assisted reproduction (5).

Circular RNAs (circRNAs) are a class of non-coding RNAs generated by back-splicing, forming a covalently closed loop (6). They are widely expressed in mammals, and many circRNAs have been identified (7). Recently, circRNAs were revealed to be modulators in numerous physiological and pathological processes. Specifically, circRNA-9119 was reported to regulate the development of ER in dairy goat by sponging 
Table I. General characteristics of the patients in this study.

\begin{tabular}{lccc}
\hline Terms & Case group $(\mathrm{n}=24)$ & Control group $(\mathrm{n}=24)$ & P-value \\
\hline Age, years & $32.75 \pm 0.40$ & $31.46 \pm 0.84$ & 0.17 \\
Infertility years & $5.75 \pm 0.45$ & $5.125 \pm 0.45$ & 0.33 \\
BMI, $\mathrm{kg} / \mathrm{m}^{2}$ & $21.21 \pm 0.48$ & $20.93 \pm 0.32$ & 0.62 \\
Age of menarche, years & $13.83 \pm 0.24$ & $13.17 \pm 0.32$ & 0.10 \\
Menstrual cycle, days & $51.78 \pm 16.28$ & $36.22 \pm 4.39$ & 0.37 \\
Menstrual duration & $5.67 \pm 0.44$ & $5.56 \pm 0.53$ & 0.87
\end{tabular}

Differences between the two groups were compared using Student's t-test. $\mathrm{P}<0.05$ indicated statistical significance. BMI, body mass index.

miR-26a (8). However, the role of circRNAs in human infertility remains unclear and requires further investigation.

Acupuncture and moxibustion, which are important in traditional Chinese medicine, have gained increased popularity for treating infertility. A prevalence study that included eight community and academic infertility practices in the United States reported that $22 \%$ of their patients had tried acupuncture treatment for infertility (9). Acupuncture combined with embryo transfer can increase pregnancy and live birth rates in women undergoing in vitro fertilization. The pregnancy rate was $42.5 \%$ (34 out of 80 patients) in the acupuncture treatment patients, while only $26.3 \%$ ( 21 of 80 patients) pregnancy rate in patients without acupuncture (10). However, the molecular mechanism of acupuncture in treating infertility has not been determined.

The aim of the present study was to verify that acupuncture and moxibustion can be used to treat infertility by altering the expression of circRNAs. CircRNA sequencing and reverse transcription-quantitative (RT-q)PCR were conducted to examine circRNA differences between patients treated with and those treated without acupuncture and moxibustion. Then, the functions and effects of circRNAs were analyzed by bioinformatics methods, including Gene Ontology (GO) analysis and pathway analysis.

\section{Materials and methods}

Patients. From January 2016 to June 2017, 48 infertile women of childbirth age treated at the Reproductive Medical Center of Nanjing Jinling Hospital, were recruited into this study. These women suffered from various diseases that lead to infertility and were planning on receiving IVF-ET. To prepare the endometrium for IVF-ET, the women were randomly assigned into two groups: One received a regular endometrial preparation program and the other received acupuncture and moxibustion in addition to the regular endometrial preparation program, with 24 women in each group. All women were monitored for the development of follicles by transvaginal ultrasound. Endometrial samples were extracted on day luteinizing hormone $(\mathrm{LH})+7$. Acupuncture was applied at the points of guanyuan, zigong, zusanli, sanyinjiao, shenshu, and ciliao. Acupuncture and moxibustion were administered starting the first day of the two natural menstrual cycles before the in vitro embryo transfer cycle up to the implantation day, one time on every other day. Patients received acupuncture for $1 \mathrm{~h}$ at a time, including half an hour on the front and half an hour on the back. The clinical characteristics of the women in this study are presented in Table I. The follow-up data revealed that the pregnancy rate in the women treated with acupuncture and moxibustion (58.33\%) was higher than that in the women who did not receive this treatment $(41.67 \%)$. The present study was approved by the Ethics Committee of Nanjing Medical University (No. (2014)204). All women provided written informed consent.

RNA extraction, library construction, and circRNA sequencing analysis. Six women in each group were used for circRNA sequencing, and 24 women in each group were used for real time PCR. Total RNA of endometrial samples from the case group and control group were extracted using TRIzol (Invitrogen; Thermo Fisher Scientific, Inc.), according to the manufacturer's instructions. RNA quality and quantity were evaluated with a Nanodrop2000 (Thermo Fisher Scientific, Inc.). Qualified RNA was processed for library construction using a TruSeq Stranded mRNA sample preparation kit and sequenced on a Hiseq ${ }^{\mathrm{TM}} 2500$ platform (both from Illumina, Inc.) with a paired-end $150 \mathrm{bp}$ read run.

Bioinformatics analysis of sequencing data. The FASTQ files generated by RNA sequencing were aligned to the USCS hg38.0 Homo sapiens reference genome with HISAT2 (11). The abundance of gene expression was calculated using fragments per kilobase of exon per million fragments mapped (FPKM). Differentially expressed (DE) mRNAs and circRNAs were screened using the DeSeq package (12) and EBSeq package (13) based on $\mid \log _{2}$ fold change (FC)|>1 and a false discovery rate (FDR) $<0.05$. Functional and pathway enrichment analyses for DEmRNAs and for the host genes of DEcircRNAs were predicted using the DAVID and GO Consortium platforms with a threshold of FDR $<0.05$.

miRNA prediction and construction of the CeRNA network. The miRNA candidates that target the DEGs were predicted using miRanda and RNAhybrid $(14,15)$. The predicted diameters were set to energy $<-30$ and score $>160$ for miRanda prediction and energy <-30 for RNA hybrid prediction. Only the overlapped miRNAs predicted by the two databases were retained for further analysis. Cytoscape V3.0 (https://cytoscape.org) was used to construct the CeRNA network.

RT-qPCR for validation of DEmRNAs and DEcircRNAs. Total RNA of endometrial samples from 24 women in each group 
Table II. The primers used for real-time PCR.

\begin{tabular}{|c|c|}
\hline Name & Sequence \\
\hline circ-RP11-446H18.5-F & 5'-CCTGCCTTCCCTAGATATTGTCCT-3' \\
\hline circ-RP11-446H18.5-R & 5'-TCСТAATCTAGCCTCTCCTGGG-3' \\
\hline circ-MIB1-F & 5-AGTATCCAAGTGGCAATAGGCA-3' \\
\hline circ-MIB1-R & 5'-TAGTAATTCGGTAAAAGCGATGTCT-3' \\
\hline circ-MLH3-F & 5'-TGAGGACATTCAGGCTGCTT-3' \\
\hline circ-MLH3-R & 5'-ATGGTAGGTAGAAAGATGGTGAGA-3' \\
\hline circ-TET2-F & 5'-TGTCAAGACTCAATATGATTTCCCA-3' \\
\hline circ-TET2-R & 5'-GGCAAATGGGAGGTGATGGT-3' \\
\hline circ-SFMBT2-F & 5'-AATCCCTTATTGATGCTGCCA-3' \\
\hline circ-SFMBT2-R & 5'-TCGAACCAGTCAAGTCACGTATG-3' \\
\hline circ-RSF1-F & 5'-AAGGAGGTCAACAAGAACAAGGA-3' \\
\hline circ-RSF1-R & 5'-CATCAGAGGAGGGCGAAGG-3' \\
\hline circ-BABAM1-F & 5'-AGATGAGTTGGAAGCCAGCAGA-3' \\
\hline circ-BABAM1-R & 5'-TCCGTCAAGGAGAACTGGGG-3' \\
\hline circ-BACH1-F & 5'-AATCAGAAATTGAGAAGCTGGTTG-3' \\
\hline circ-BACH1-R & 5'-GTTGTCGGGAAGTTCAGTGGA-3' \\
\hline circ-LPAR1-F & 5'-TGTAATTTCACAGCCCCAGGT-3' \\
\hline circ-LPAR1-R & 5'-CAGATCGAAGTCATGCTAGGAGA-3' \\
\hline
\end{tabular}

F, forward; R, reverse.

Table III. The top five (ranked by $-\log _{10} \mathrm{P}$-value) $\mathrm{BP}$ terms, $\mathrm{CC}$ terms, and MF terms.

\begin{tabular}{|c|c|c|c|}
\hline GO ID & $-\log _{10}(\mathrm{P}$-value $)$ & Description & GO terms \\
\hline GO:0016568 & 6.1914023 & Chromatin modification & $\mathrm{BP}$ \\
\hline GO:0006990 & 3.9159859 & $\begin{array}{l}\text { Positive regulation of transcription from RNA polymerase II } \\
\text { promoter involved in unfolded protein response }\end{array}$ & $\mathrm{BP}$ \\
\hline GO:0035511 & 3.6954187 & Oxidative DNA demethylation & $\mathrm{BP}$ \\
\hline GO:0061418 & 3.5660861 & $\begin{array}{l}\text { Regulation of transcription from RNA polymerase II promoter in } \\
\text { response to hypoxia }\end{array}$ & $\mathrm{BP}$ \\
\hline GO:0051150 & 3.5206085 & Regulation of smooth muscle cell differentiation & $\mathrm{BP}$ \\
\hline GO:0005654 & 4.7545709 & Nucleoplasm & $\mathrm{CC}$ \\
\hline GO:0005730 & 3.212923 & Nucleolus & $\mathrm{CC}$ \\
\hline GO:0005634 & 2.7622145 & Nucleus & $\mathrm{CC}$ \\
\hline GO:0000123 & 2.3794772 & Histone acetyltransferase complex & $\mathrm{CC}$ \\
\hline GO:0005642 & 2.0758729 & Annulate lamellae & $\mathrm{CC}$ \\
\hline GO:0070579 & 3.9552169 & Methylcytosine dioxygenase activity & MF \\
\hline GO:0003682 & 3.0894796 & Chromatin binding & MF \\
\hline GO:0008270 & 3.0872473 & Zinc ion binding & MF \\
\hline GO:0042393 & 2.8177814 & Histone binding & MF \\
\hline GO:0005515 & 2.8066047 & Protein binding & MF \\
\hline
\end{tabular}

GO, Gene Ontology; BP, biological process; CC, cellular component; $\mathrm{MF}$, molecular function.

were extracted using TRIzol. The concentration and purity of RNA were assessed using a microspectrophotometer (Tiangen Biotech Co., Ltd.). First-strand cDNA was synthesized using the RevertAid First Strand cDNA synthesis kit (Thermo Fisher Scientific, Inc.). FastStart Universal SYBR Green Master mix was used to amplify cDNA on the QuantStudio 6 Flex Real-Time PCR System (Thermo Fisher Scientific, Inc.). The primers are presented in Table II. The $2^{-\Delta \Delta \mathrm{Cq}}$ method was used to determine the relative gene expression with the internal control of B2M (16). 
Table IV. The significantly enriched KEGG pathways [ranked by $-\log _{10}(\mathrm{P}$-value $)$ ].

\begin{tabular}{lll}
\hline PathwayID & $-\log _{10}($ P-value $)$ & \multicolumn{1}{c}{ Pathway term } \\
\hline PATH:04141 & 3.3411215 & Protein processing in endoplasmic reticulum \\
PATH:01220 & 1.9245391 & Degradation of aromatic compounds \\
PATH:05131 & 1.6033722 & Shigellosis \\
PATH:04150 & 1.5776843 & mTOR signaling pathway \\
PATH:05100 & 1.4296001 & Bacterial invasion of epithelial cells \\
PATH:05215 & 1.3156425 & Prostate cancer
\end{tabular}

KEGG, Kyoto Encyclopedia of Genes and Genomes.

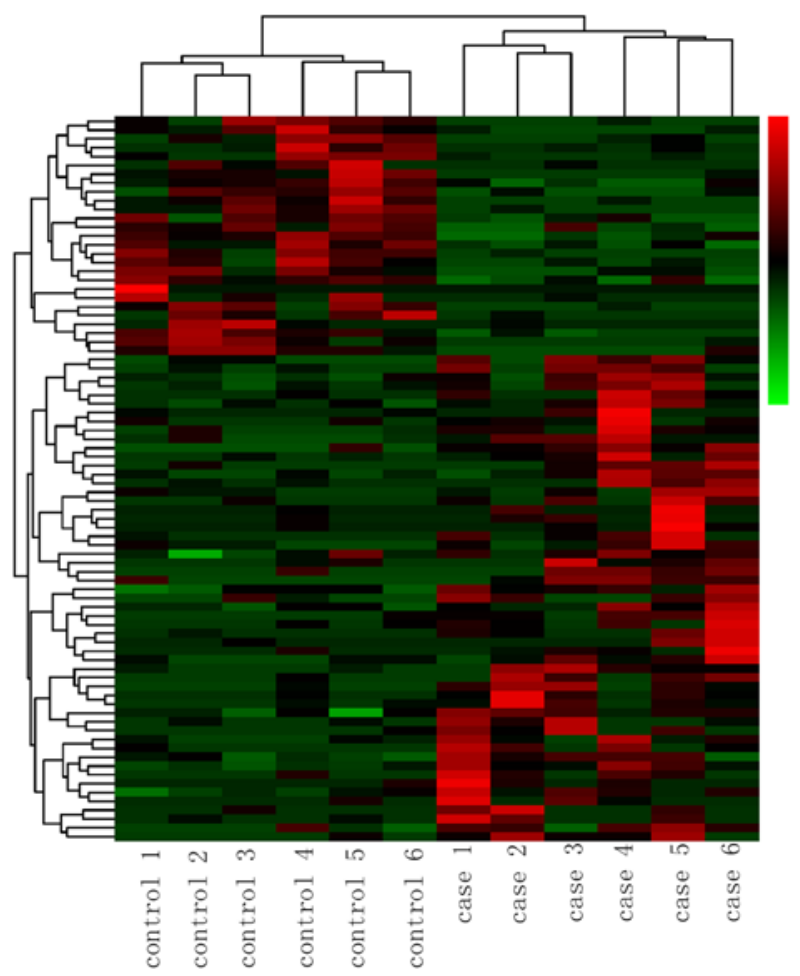

Figure 1. Cluster heatmap. The differential expression profile of circRNAs between the two study groups and the homogeneity within each group are revealed based on a hierarchical clustering analysis.

Statistical analysis. Real-time PCRs were repeated in triplicate. Data are expressed as the mean \pm standard deviation (SD) and compared using Student's t test. $\mathrm{P}<0.05$ was defined as the level of statistical significance.

\section{Results}

Several circRNAs are differentially expressed after acupuncture and moxibustion. Using high-throughput sequencing, 2,653 circRNAs were identified, and 86 circRNAs were significantly differentially expressed ( $\log 2 \mathrm{FCl}>1, \mathrm{FDR}<0.05)$ between the acupuncture group and the control group (Fig. 1). Moreover, among the DEcircRNAs, 57 circRNAs were upregulated, and 29 were downregulated.

GO and pathway analysis of differential circRNAs. The roles of circRNAs were investigated by GO analysis within three

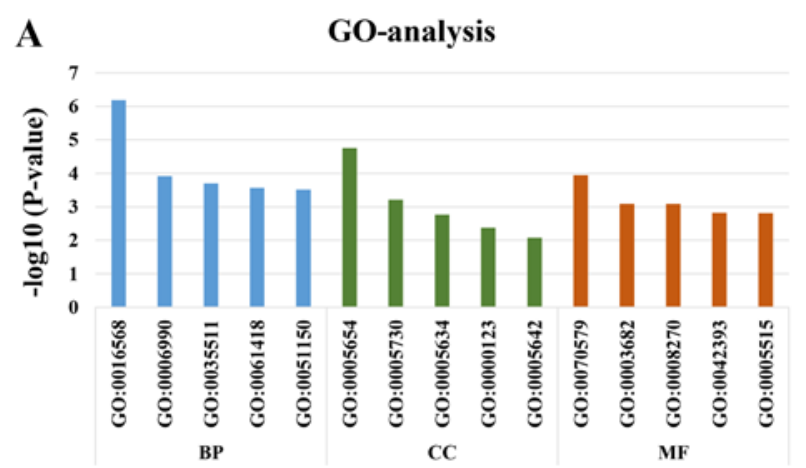

B

Pathway-analysis

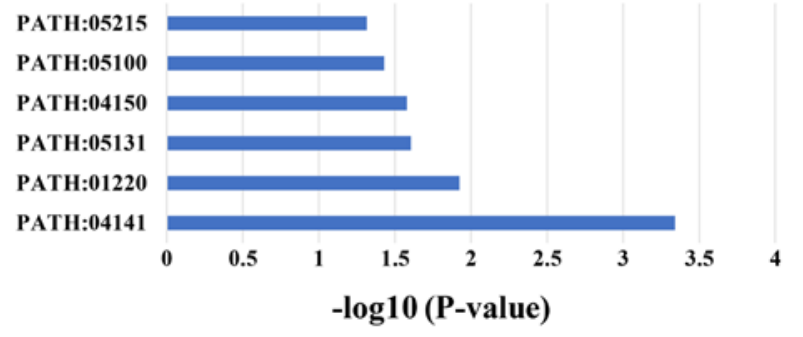

Figure 2. GO and pathways related to differentially expressed circRNAs. (A) The top 15 terms of BP, CC, and MF. (B) The significantly enriched pathways $\left(-\log _{10} \mathrm{P}>1.301\right)$. GO, Gene Ontology; BP, biological process; $\mathrm{CC}$, cellular component; MF, molecular function.

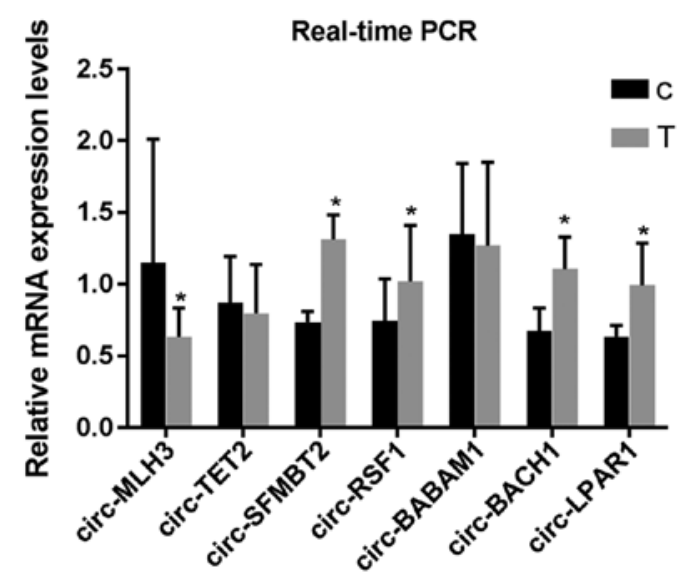

Figure 3. Relative expression levels of the circRNAs. Expression of seven differentially expressed circRNAs was validated by reverse transcriptionquantitative PCR. $n=24,{ }^{*} \mathrm{P}<0.05$ vs. respective C. C, control; $\mathrm{T}$, tumor. 


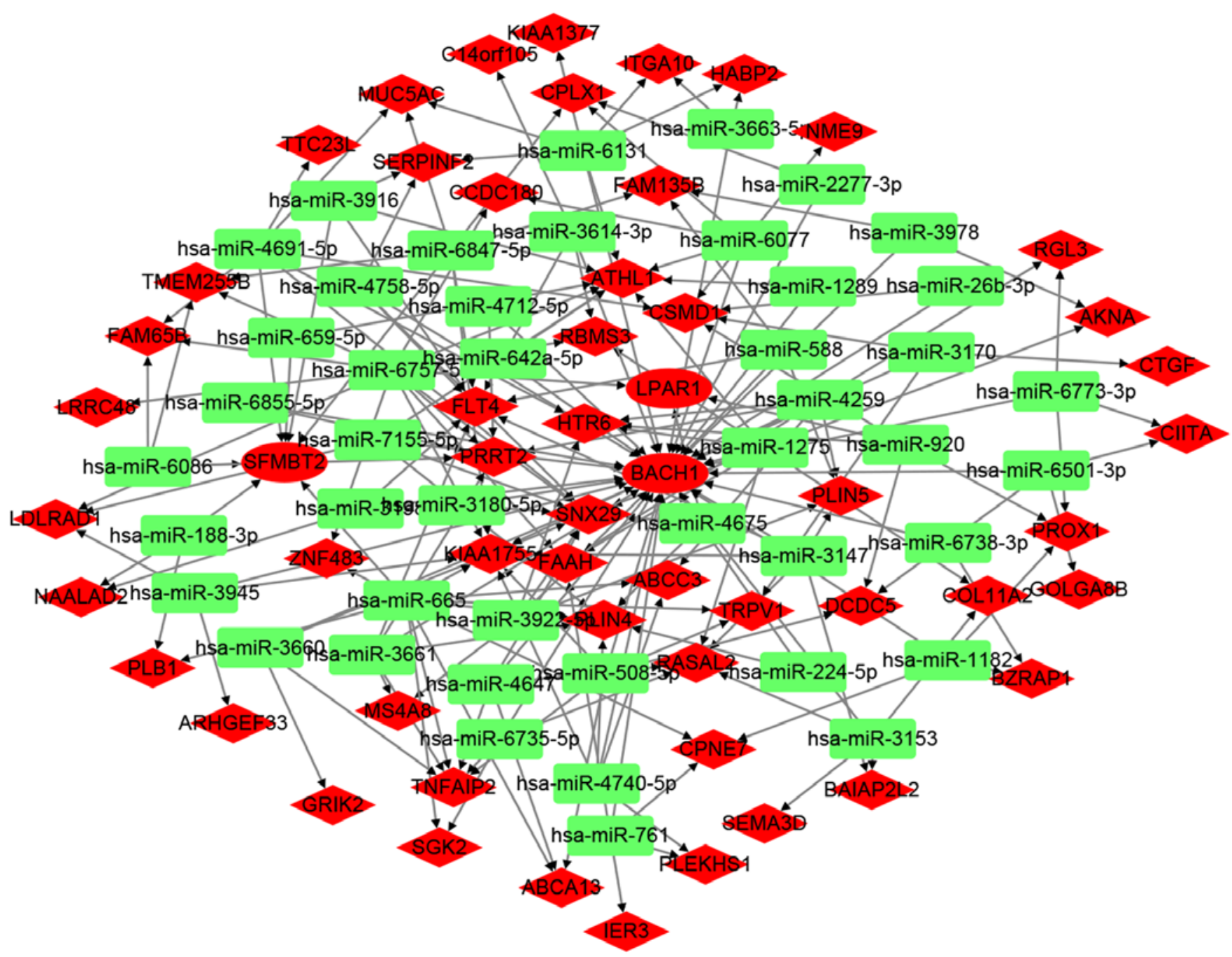

Figure 4. circRNA-miR-mRNA interaction network. circBACH1 was associated with 35 miRs, circSFMBT2 with 7 miRs, and circLPAR1 with 3 miRs. miRs, microRNAs.

different domains [biological process (BP), cellular component (CC), and molecular function (MF)]. The top five terms of each domain, ranked by $-\log _{10}(\mathrm{P}$-value), are presented in Fig. 2A and Table III. The identified BP terms were chromatin modification, positive regulation of transcription from RNA polymerase II promoter involved in unfolded protein response, oxidative DNA demethylation, regulation of transcription from RNA polymerase II promoter in response to hypoxia, and regulation of smooth muscle cell differentiation. The identified $\mathrm{CC}$ terms were nucleoplasm, nucleolus, nucleus, histone acetyltransferase complex, and annulate lamellae. The identified MF terms were methylcytosine dioxygenase activity, chromatin binding, zinc ion binding, histone binding, and protein binding. The significantly enriched KEGG pathways, ranked by $-\log _{10}(\mathrm{P}$-value), are shown in Fig. $2 \mathrm{~B}$ and Table IV. The identified pathways were protein processing in endoplasmic reticulum, degradation of aromatic compounds, shigellosis, mTOR signaling pathway, bacterial invasion of epithelial cells, and prostate cancer.

circRNAs associated with infertility are differentially expressed after acupuncture and moxibustion. Seven DEcircRNAs (circ-SFMBT2, circ-MLH3, circ-RSF1, circ-BABAM1,
circ-TET2, circ-BACH1, and circ-LPAR1) associated with infertility were selected to verify the sequencing results by RT-qPCR. The relative expression levels of the seven circRNAs are presented in Fig.3. The variation tendencies of circ-SFMBT2, circ-MLH3, circ-RSF1, circ-BACH1, and circ-LPAR1, but not those of circ-BABAM1 and circ-TET2, were in accord with those observed in high-throughput sequencing. The expression levels of circ-SFMBT2, circ-BACH1, circ-RSF1, and circ-LPAR1 were significantly upregulated and that of circ-MLH3 was downregulated in samples from patients treated with acupuncture and moxibustion compared to levels in the control group.

Construction of the circRNA-miR-mRNA interaction network. To evaluate the potential functions of the DE circRNAs, upregulated circRNAs were selected, and a circRNA-miR-mRNA interaction network was constructed (Fig. 4). circBACH1 was associated with 35 downregulated miRs, followed by circSFMBT2 with 7 downregulated miRs and circLPAR1 with 3 downregulated miRs, indicating that circBACH1 may play crucial roles in the network. Additionally, six genes (ATHL1, HTR6, KIAA1755, PLIN4, PRRT2, SNX29) were related to all three circRNAs. 


\section{Discussion}

With the rapid development of high-throughput sequencing technology, the roles of many circRNAs are being clarified. circRNAs have been confirmed to be vital modulators in a large number of physiological and pathological processes. circFBLIM1 was reported to promote hepatocellular cancer progression by sponging miR-346 (7). circNT5E was reported to promote glioblastoma tumorigenesis (17). However, studies on the functions and biological characteristics have mainly involved cancers, and there are few studies on circRNAs regarding the endometrium. Although circRNA-9119 was confirmed to regulate the development of ER in dairy goat (8), studies on the effects of circRNAs on human ER remain very limited.

Using high-throughput RNA sequencing, 2,653 circRNAs were identified. Among the 86 DEcircRNAs $(\mid \log 2 \mathrm{FCl}>1$, FDR $<0.05), 57$ were upregulated and 29 were downregulated between the acupuncture group and the control group. Nine circRNAs were selected for further verification, and three (circ-SFMBT2, circ-BACH1, and circ-LPAR1) were differentially expressed in the acupuncture and moxibustion group.

The potential regulatory role of circRNAs was investigated by conducting KEGG and GO analyses. The significantly enriched terms embryonic digestive tract morphogenesis (GO:0048557) and regulation of smooth muscle cell differentiation (GO:0051150) indicate the possibility of altering the endometrial thickness and influencing ER. Notably, estrogen and progestogen are two important hormones involved in regulating the growth and development of the endometrium $(18,19)$. Estrogen and progestogen are both aromatic compounds, and the degradation of the aromatic compound pathway (PATH:01220) was significantly enriched. Analogously, protein processing in the endoplasmic reticulum pathway (PATH:04141) was also significantly enriched, and the progestogen receptor has been reported to influence the ER (20). Moreover mTOR was reported to play a part in the tumorigenesis and development of endometrial carcinoma (21), and notably, the mTOR signaling pathway (PATH:04150) was revealed to be significantly enriched. Thus, the degradation of the aromatic compound pathway, protein processing in the endoplasmic reticulum pathway, and the mTOR signaling pathway are most likely involved in the regulation of ER.

Six genes (ATHL1, HTR6, KIAA1755, PLIN4, PRRT2, SNX29) were associated with all three differential circRNAs (circ-SFMBT2, circ-BACH1, and circ-LPAR1). In particular, circBACH1 was associated with the most genes, indicating that it may play a dominant role in the regulatory network as a competitively endogenous RNA (ceRNA) and can serve as a biomarker of ER. Furthermore, PLIN4 was revealed to be associated with steroids (22), which included estrogen, indicating that PLN4 may be important for ER. In future studies, PLN4, can be assessed as a potential target for new medicines and treatments. Our results indicated that acupuncture and moxibustion affect ER via regulation of the expression of circRNAs. circBACH1 may influence ER as a ceRNA of miRs, and PLN4 may play a dominate role in ER.

\section{Acknowledgements}

Not applicable.

\section{Funding}

The present study was supported by the National Natural Science Foundation of China (81473767, 81873371, 81804179, 81403477, 81603674 and 81403481), the Jiangsu Provincial Science and Technology Project of the Traditional Chinese Medicine Bureau (YB2017005), and the Special Foundation for Clinical Medical Research of the Chinese Medical Association-Research and Development Project of Young Physicians of Reproductive Medicine (17020350704).

\section{Availability of data and materials}

The datasets used and analyzed during the present study are available from the corresponding author on reasonable request.

\section{Authors' contributions}

JS and LC designed the experiments. JS, LC, JC, XJ, YM, QL, LX, YG and YX performed the experiments. JS, JC and XJ analyzed the data; and JS, LC and JC prepared the manuscript. All authors read and approved the manuscript and agree to be accountable for all aspects of the research in ensuring that the accuracy or integrity of any part of the work are appropriately investigated and resolved.

\section{Ethics approval and consent to participate}

The present study was approved by the Ethics Committee of Nanjing Medical University (no. (2014)204). All patients provided written informed consent.

\section{Patient consent for publication}

Not applicable.

\section{Competing interests}

The authors declare that they have no competing interests.

\section{References}

1. Inhorn MC and Patrizio P: Infertility around the globe: New thinking on gender, reproductive technologies and global movements in the 21st century. Hum Reprod Update 21: 411-426, 2015.

2. Pandian Z, Gibreel A and Bhattacharya S: In vitro fertilisation for unexplained subfertility. Cochrane Database Syst Rev: CD003357, 2015.

3. Cha J, Sun X and Dey SK: Mechanisms of implantation: Strategies for successful pregnancy. Nat Med 18: 1754-1767, 2012.

4. Wang $\mathrm{H}$ and Dey SK: Roadmap to embryo implantation: Clues from mouse models. Nat Rev Genet 7: 185-199, 2006.

5. Teh WT, McBain J and Rogers P: What is the contribution of embryo-endometrial asynchrony to implantation failure? J Assist Reprod Genet 33: 1419-1430, 2016.

6. Hu W, Han Q,ZZhao L and Wang L: Circular RNA circRNA_15698 aggravates the extracellular matrix of diabetic nephropathy mesangial cells via miR-185/TGF- $\beta 1$. J Cell Physiol 234: 1469-1476, 2019.

7. Bai N, Peng E, Qiu X, Lyu N, Zhang Z, Tao Y, Li X and Wang Z: circFBLIM1 act as a ceRNA to promote hepatocellular cancer progression by sponging miR-346. J Exp Clin Cancer Res 37: 172,2018

8. Zhang L, Liu X, Che S, Cui J, Liu Y, An X, Cao B and Song Y: CircRNA-9119 regulates the expression of prostaglandin-endoperoxide synthase 2 (PTGS2) by sponging miR-26a in the endometrial epithelial cells of dairy goat. Reprod Fertil Dev 30: 1759-1769, 2018. 
9. Smith J, Eisenberg M, Millstein S, Nachtigall RD, Shindel AW, Wing H, Cedars M, Pasch L and Katz PP; Infertility Outcomes Program Project Group: The use of complementary and alternative fertility treatment in couples seeking fertility care: Data from a prospective cohort in the United States. Fertil Steril 93: 2169-2174, 2010

10. Paulus WE, Zhang M, Strehler E, El-Danasouri I and Sterzik K: Influence of acupuncture on the pregnancy rate in patients who undergo assisted reproduction therapy. Feril Steril 77: 721-724, 2002.

11. Kim D, Langmead B and Salzberg SL: HISAT: A fast spliced aligner with low memory requirements. Nat Methods 12: 357-360, 2015.

12. Wang L, Feng Z, Wang X, Wang X and Zhang X: DEGseq: An $R$ package for identifying differentially expressed genes from RNA-seq data. Bioinformatics 26: 136-138, 2010.

13. Leng N, Dawson JA, Thomson JA, Ruotti V, Rissman AI, Smits BM, Haag JD, Gould MN, Stewart RM and Kendziorski C: EBSeq: An empirical Bayes hierarchical model for inference in RNA-seq experiments. Bioinformatics 29: 1035-1043, 2013.

14. Krüger $\mathbf{J}$ and Rehmsmeier M: RNAhybrid: microRNA target prediction easy, fast and flexible. Nucleic Acids Res 34 (Web Server issue): W451-W454, 2006.

15. Rehmsmeier M, Steffen P, Hochsmann M and Giegerich R: Fast and effective prediction of microRNA/target duplexes. RNA 10: $1507-1517,2004$
16. Livak KJ and Schmittgen TD: Analysis of relative gene expression data using real-time quantitative PCR and the 2(-Delta Delta C(T)) method. Methods 25: 402-408, 2001.

17. Wang R, Zhang S, Chen X, Li N, Li J, Jia R, Pan Y and Liang H: CircNT5E acts as a sponge of microRNA-422a to promote glioblastoma tumorigenesis. Cancer Res 78: 4812-4825, 2018.

18. Vercellini P, Buggio L, Berlanda N, Barbara G, Somigliana E and Bosari S: Estrogen-progestins and progestins for the management of endometriosis. Fertil Steril 106: 1552-1571.e2, 2016.

19. Vujović S, Ivovic M, Tančić-Gajić M, Marina L, Ljubic A, Dragojević-Dikić S and Genazzani AR: Endometrium receptivity in premature ovarian insufficiency-how to improve fertility rate and predict diseases? Gynecol Endocrinol 34: 1011-1015, 2018.

20. Pei T, Liu C, Liu T, Xiao L, Luo B, Tan J, Li X, Zhou G, Duan C and Huang W: miR-194-3p represses the progesterone receptor and decidualization in eutopic endometrium from women with endometriosis. Endocrinology 159: 2554-2562, 2018.

21. Du Y, Wang L, Chen S, Liu Y and Zhao Y: lncRNA DLEU1 contributes to tumorigenesis and development of endometrial carcinoma by targeting mTOR. Mol Carcinog 57: 1191-1200, 2018.

22. Kraemer F, Khor V, Shen W and Azhar S: Cholesterol ester droplets and steroidogenesis. Mol Cell Endocrinol 371: 15-19, 2013. 